Kardiologe 2018 $\cdot 12: 53-55$

https://doi.org/10.1007/s12181-017-0221-6

Online publiziert: 24. Januar 2018

(c) Deutsche Gesellschaft für Kardiologie Herz- und Kreislaufforschung e.V. Published by Springer Medizin Verlag $\mathrm{GmbH}$, ein Teil von Springer Nature - all rights reserved 2018

CrossMark

H. M. Nef' $\cdot$ M. Abdel-Wahab 2 . S. Achenbach ${ }^{3} \cdot$ M. Joner ${ }^{4} \cdot$ B. Levenson ${ }^{5} \cdot$ J. Mehilli ${ }^{6}$. H. Möllmann ${ }^{7} \cdot$ H. Thiele $^{8} \cdot$ R. Zahn ${ }^{9} \cdot$ T. Zeus $^{10} \cdot$ A. Elsässer ${ }^{11}$

${ }^{1}$ Medizinische Klinik I, Kardiologie und Angiologie, Universitätsklinikum Gießen und Marburg, Gießen, Deutschland

${ }^{2}$ Herzzentrum, Segeberger Kliniken, Bad Segeberg, Deutschland

${ }^{3}$ Medizinische Klinik 2, Universitätsklinikum Erlangen, Friedrich-Alexander-Universität ErlangenNürnberg, Erlangen, Deutschland

${ }^{4}$ Kardiologie, Herzzentrum München, München, Deutschland

${ }^{5}$ Kardiologische Gemeinschaftspraxis und Herzkatheterlabor, Berlin, Deutschland

${ }^{6}$ Klinikum der Universität München, Medizinische Klinik und Poliklinik I, DZHK (Deutsches Zentrum für Herz-Kreislauf-Forschung e.V.), Standort München, Ludwig-Maximilian-Universität, München, Deutschland

${ }^{7}$ Klinik für Innere Medizin I, St.-Johannes-Hospital, Dortmund, Deutschland

${ }^{8}$ Herzzentrum, Klinik für Innere Medizin/Kardiologie Leipzig, Universität Leipzig, Leipzig, Deutschland

${ }^{9}$ Medizinische Klinik B, Klinikum der Stadt Ludwigshafen, Ludwigshafen, Deutschland

${ }^{10} \mathrm{Klinik}$ für Kardiologie, Pneumologie und Angiologie, Universitätsklinikum Düsseldorf, Düsseldorf, Deutschland

${ }^{11}$ Herzzentrum, Universität Oldenburg, Oldenburg, Deutschland

\title{
Correction:
}

\section{Medikamentenfreisetzende Koronarstents/-scaffolds und medikamentenbeschichtete Ballonkatheter}

\section{Positionspapier der Arbeitsgruppe Interventionelle Kardiologie (AGIK) der Deutschen Gesellschaft für Kardiologie - Herz- und Kreislaufforschung e. V.}

\section{Correction:}

\section{Kardiologe 2017}

https://doi.org/10.1007/s12181-017-

0202-9

In diesem Beitrag ist leider die • Tab. 1 fehlerhaft dargestellt.

Ferner sind in - Tab. 6 in der vorletzten Zeile der Herstellername (Biosensors) und der Name des Ballonsystems (Biostream) vertauscht.

Wir bitten um Beachtung der korrigierten Tabellen (•Tab. 1 und • Tab. 6).

\section{Korrespondenzadresse}

\section{Prof. Dr. H. M. Nef}

Medizinische Klinik I, Kardiologie und Angiologie, Universitätsklinikum Gießen und Marburg

Klinikstr. 33, 33539 Gießen, Deutschland holger.nef@innere.med.uni-giessen.de

Die Online-Version des Originalartikels ist unter https://doi.org/10.1007/s12181-017-0202-9zu finden. 


\section{Correction}

Tab. 1 Medikamentenbeschichtete Stents mit CE-Zulassung, Verfügbarkeit in Deutschland und mindestens einer randomisierten publizierten Studie

\begin{tabular}{|c|c|c|c|c|c|c|c|}
\hline Stentsystem & Hersteller & Medikament & Trägerbeschichtung & Stentgerüst & $\begin{array}{l}\text { Endpunkt } \\
\text { klinisch }\end{array}$ & $\begin{array}{l}\text { Endpunkt angio- } \\
\text { graphisch }\end{array}$ & Literatur \\
\hline \multicolumn{8}{|c|}{ DES mit permanenter Polymerbeschichtung } \\
\hline $\begin{array}{l}\text { Taxus Express/ } \\
\text { Liberté }\end{array}$ & $\begin{array}{l}\text { Boston Scien- } \\
\text { tific }\end{array}$ & Paclitaxel & $\begin{array}{l}\text { Poly-Styren-b- } \\
\text { isobutylen-b-styren } \\
\text { (SIBS) }\end{array}$ & Stainless Steel & ++ & ++ & {$[8,19-23]$} \\
\hline Xience & $\begin{array}{l}\text { Abbott Vascu- } \\
\text { lar }\end{array}$ & Everolimus & $\begin{array}{l}\text { Poly-Vinyliden-Fluorid- } \\
\text { Hexafluoro-Propylen } \\
\text { (PVDF-HFP) }\end{array}$ & $\mathrm{CoCr}$ & +++ & +++ & $\begin{array}{l}{[8,20,24,} \\
25]\end{array}$ \\
\hline $\begin{array}{l}\text { Promus } \\
\text { Promus Element }\end{array}$ & $\begin{array}{l}\text { Boston Scien- } \\
\text { tific }\end{array}$ & Everolimus & $\begin{array}{l}\text { Poly-Vinyliden-Fluorid- } \\
\text { Hexafluoro-Propylen } \\
\text { (PVDF-HFP) }\end{array}$ & $\begin{array}{l}\mathrm{CoCr} \\
\text { Platin-Chrom }\end{array}$ & $\begin{array}{l}+++ \\
++\end{array}$ & $\begin{array}{l}+++ \\
++\end{array}$ & {$[8,24,26]$} \\
\hline Endeavor & Medtronic & Zotarolimus & $\begin{array}{l}\text { Phosphorylcholin (ABT } \\
578 \text { ) }\end{array}$ & $\mathrm{CoCr}$ & ++ & ++ & {$[21,27,28]$} \\
\hline $\begin{array}{l}\text { Resolute Integri- } \\
\text { ty } \\
\text { Resolute Onyx }\end{array}$ & Medtronic & Zotarolimus & 3 Komponenten Biolinx & $\begin{array}{l}\mathrm{CoCr} \\
\text { Platinum } \\
\text { Iridium }\end{array}$ & $\begin{array}{l}++ \\
+\end{array}$ & $\begin{array}{l}++ \\
+\end{array}$ & {$[28,29]$} \\
\hline \multicolumn{8}{|c|}{ DES mit biodegradierbarer Polymerbeschichtung } \\
\hline $\begin{array}{l}\text { Biomatrix } \\
\text { Neoflex } \\
\text { Biomatrix alpha }\end{array}$ & Biosensors & Biolimus A9 & Resorbierbares Polymer & $\begin{array}{l}\text { Edelstahl } \\
\mathrm{CoCr}\end{array}$ & + & - & {$[30,31]$} \\
\hline Orsiro/Synsiro & Biotronik & Sirolimus & Biolute PLLA & $\mathrm{CoCr}$ & + & + & [32] \\
\hline Synergy & $\begin{array}{l}\text { Boston Scien- } \\
\text { tific }\end{array}$ & Everolimus & Abluminal PLGA & $\begin{array}{l}\text { Platinum } \\
\text { Chrom }\end{array}$ & + & + & [33] \\
\hline Ultimaster & Terumo & Sirolimus & $\begin{array}{l}\text { Abluminal Poly (DL-lac- } \\
\text { tide-co-caprolactone) }\end{array}$ & $\mathrm{CoCr}$ & + & + & [34] \\
\hline \multicolumn{8}{|l|}{ Polymerfreie DES } \\
\hline Biofreedom & Biosensors & Biolimus A9 & $\begin{array}{l}\text { Abluminale mikroporö- } \\
\text { se Oberfläche }\end{array}$ & Stainless Steel & + & + & {$[35]$} \\
\hline Coroflex ISAR & Braun & $\begin{array}{l}\text { Sirolimus/ } \\
\text { Probucol }\end{array}$ & N. v. & $\mathrm{CoCr}$ & + & + & [15] \\
\hline Yukon Choice & Translumina & Sirolimus & $\begin{array}{l}\text { Abluminale mikroporö- } \\
\text { se Oberfläche }\end{array}$ & Stainless Steel & + & + & [36] \\
\hline \multicolumn{8}{|c|}{ DES mit anderen Technologien } \\
\hline Combo & $\begin{array}{l}\text { Orbus-Neich } \\
\text { Medical }\end{array}$ & EPC+Sirolimus & $\begin{array}{l}\text { Abluminal biodegra- } \\
\text { dierbares Polymer und } \\
\text { luminale CD34-AK }\end{array}$ & Stainless Steel & + & + & [17] \\
\hline
\end{tabular}




\begin{tabular}{|c|c|c|c|c|c|}
\hline Ballonsystem & Hersteller & $\begin{array}{l}\text { Zusatz zur Be- } \\
\text { schichtung }\end{array}$ & Wirksubstanz & $\begin{array}{l}\text { Angiographische } \\
\text { Endpunktstudien }\end{array}$ & Ausgewählte Literatur \\
\hline SeQuent Please & B. Braun & $\begin{array}{l}\text { Kontrastmittel } \\
\text { (Iopromid) }\end{array}$ & $\begin{array}{l}\text { Paclitaxel } \\
3 \mu \mathrm{g} / \mathrm{mm}^{2}\end{array}$ & ++ ISR, DN, Bif, SVD & {$[143,148-157]$} \\
\hline Pantera Lux & Biotronik & $\begin{array}{l}\text { Butyryl-trihexyl } \\
\text { Zitrat }\end{array}$ & $\begin{array}{l}\text { Paclitaxel } \\
3 \mu \mathrm{g} / \mathrm{mm}^{2}\end{array}$ & $(+)$ ISR & [158-160] \\
\hline In.Pact Falcon & Medtronic & Harnstoff & $\begin{array}{l}\text { Paclitaxel } \\
3 \mu \mathrm{g} / \mathrm{mm}^{2}\end{array}$ & $(+)$, Bif, DN & [161-163] \\
\hline Cotavance & Medrad Interventional & $\begin{array}{l}\text { Kontrastmittel } \\
\text { (Ultravist) }\end{array}$ & $\begin{array}{l}\text { Paclitaxel } \\
3 \mu \mathrm{g} / \mathrm{mm}^{2}\end{array}$ & - & N.a. \\
\hline Dior II & Eurocor & Schellack & $\begin{array}{l}\text { Paclitaxel } \\
3 \mu \mathrm{g} / \mathrm{mm}^{2}\end{array}$ & (+) Bif, DN & [164-166] \\
\hline Protege & Blue Medical & $\begin{array}{l}\text { Butyryl-trihexyl } \\
\text { Zitrat }\end{array}$ & $\begin{array}{l}\text { Paclitaxel } \\
3 \mu \mathrm{g} / \mathrm{mm}^{2}\end{array}$ & - & N. a. \\
\hline Elutax & AachenResonance & Kein Excipient & $\begin{array}{l}\text { Paclitaxel } \\
2 \mu \mathrm{g} / \mathrm{mm}^{2}\end{array}$ & $(+)$ ISR & [145] \\
\hline Restore DCB & Cardionovum & $\begin{array}{l}\text { Kein Excipient } \\
\text { Paclitaxel als } \\
\text { Nanokristalle }\end{array}$ & $\begin{array}{l}\text { Paclitaxel } \\
3 \mu \mathrm{g} / \mathrm{mm}^{2}\end{array}$ & $(+)$ ISR & [167] \\
\hline Danubio & Minvasys & $\begin{array}{l}\text { Butyryl-trihexyl- } \\
\text { Citrat }\end{array}$ & $\begin{array}{l}\text { Paclitaxel } \\
2,5 \mu \mathrm{g} / \mathrm{mm}^{2}\end{array}$ & (+) Bif & [168] \\
\hline Agent & Boston & Citratester & $\begin{array}{l}\text { Paclitaxel } \\
2 \mu \mathrm{g} / \mathrm{mm}^{2}\end{array}$ & - & N. a. \\
\hline Biostream & Biosensors & Shellac & $\begin{array}{l}\text { Paclitaxel } \\
3 \mu \mathrm{g} / \mathrm{mm}^{2}\end{array}$ & - & N. a. \\
\hline Moxy & Lutonix & Polysorbate & $\begin{array}{l}\text { Paclitaxel } \\
3 \mu \mathrm{g} / \mathrm{mm}^{2}\end{array}$ & (+) DN & [169] \\
\hline
\end{tabular}

